\title{
Meclofenamate elicits a nephropreventing effect in a rat model of ischemic acute kidney injury by suppressing indoxyl sulfate production and restoring renal organic anion transporters
}

\author{
This article was published in the following Dove Press journal: \\ Drug Design, Development and Therapy \\ 13 August 2014 \\ Number of times this article has been viewed
}

\section{Chika Saigo' \\ Yui Nomura' \\ Yuko Yamamoto' \\ Masataka Sagata' \\ Rika Matsunaga' \\ Hirofumi Jono ${ }^{1,2}$ \\ Kazuhiko Nishi ${ }^{3}$ \\ Hideyuki Saito ${ }^{1,2}$ \\ 'Department of Clinical \\ Pharmaceutical Sciences, Graduate School of Pharmaceutical Sciences, Kumamoto University, ${ }^{2}$ Department of Pharmacy, Kumamoto University Hospital, ${ }^{3}$ Department of Hemo- Dialysis, Kumamoto University Hospital, Kumamoto, Japan}

Correspondence: Hideyuki Saito Department of Pharmacy, Kumamoto University Hospital, I-I-I Honjo, Chuo-ku, Kumamoto,

Kumamoto 860 8556, Japan

Tel/Fax +8I 963735820

Email saitohide@fc.kuh.kumamoto-u.ac.jp
Abstract: Indoxyl sulfate (IS), a putative low-molecular weight uremic toxin, is excreted in the urine under normal kidney function, but is retained in the circulation and tissues during renal dysfunction in acute kidney injury and chronic kidney disease. IS, which is one of the most potent inducers of oxidative stress in the kidney and cardiovascular system, is enzymatically produced in the liver from indole by cytochrome P450-mediated hydroxylation to indoxyl, followed by sulfotransferase-mediated sulfate conjugation. We used rat liver S9 fraction to identify inhibitors of IS production. After testing several compounds, including phytochemical polyphenols, we identified meclofenamate as a potent inhibitor of IS production with an apparent $\mathrm{IC}_{50}$ value of $1.34 \mu \mathrm{M}$. Ischemia/reperfusion (I/R) of rat kidney caused a marked elevation in the serum IS concentration 48 hours after surgery. However, intravenous administration of meclofenamate $(10 \mathrm{mg} / \mathrm{kg})$ significantly suppressed this increase in the serum level of IS. Moreover, IS concentrations in both kidney and liver were dramatically elevated by renal I/R treatment, but this increase was blocked by meclofenamate. Serum creatinine and blood urea nitrogen were markedly elevated in rats after renal I/R treatment, but these increases were significantly restored by administration of meclofenamate. Renal expression of both basolateral membrane-localized organic anion transporters rOAT1 and rOAT3 was downregulated by I/R treatment. However, expression of rOAT1 and rOAT3 recovered after administration of meclofenamate, which is associated with the inhibition of I/R-evoked elevation of prostaglandin E2. Our results suggest that meclofenamate inhibits hepatic sulfotransferase-mediated production of IS, thereby suppressing serum and renal accumulation of IS. Meclofenamate also prevents the prostaglandin E2-dependent downregulation of rOAT1 and rOAT3 expression. In conclusion, meclofenamate was found to elicit a nephropreventive effect in ischemic acute kidney injury.

Keywords: uremic toxins, hepatic sulfotransferase, renal ischemia/reperfusion, renal tubular cell

\section{Introduction}

The Acute Kidney Injury Network (AKIN) defined acute kidney injury (AKI) as "functional and structural disorders or signs of renal damage including any defect from blood and urine test, or tissue imaging that is less than 3 months". ${ }^{1,2}$ AKI develops when the kidney damage is evoked by a decrease in renal blood flow, ie, renal ischemia from causes including low blood pressure and renal surgery, exposure to nephrotoxic chemicals and drugs, an inflammatory process in the kidney, or an obstruction of the urinary tract that blocks urine flow. ${ }^{2}$ Generally, AKI is diagnosed based on laboratory data, such as abnormal blood urea nitrogen (BUN) and serum creatinine ( $\mathrm{SCr}$ ) levels, or failure to produce sufficient amounts of urine. AKI leads to many complications, 
including metabolic acidosis, high potassium levels, changes in body fluid balance, and uremia with increased serum uremic toxins that cause oxidative stress affecting other organ functions.

Uremic toxins are defined as compounds retained as solutes in the serum that contribute to uremic syndrome (ie, deterioration of multiple biochemical and physiological functions in association with progressive renal disease), leading to a complex and variable symptomatology. 3,4 These uremic toxins accumulate in patients with chronic kidney disease (CKD), including patients with stage 5 kidney disease or end-stage renal disease. ${ }^{5,6}$ Some studies have suggested that moderate stages of AKI are independently associated with an increase in mortality. ${ }^{7-9}$ Increased mortality is not due to AKI or to direct renal complications associated with AKI but is enhanced by acute lung injury, sepsis or sympathetic nervous system dysfunctions. The precise mechanism by which AKI induces these deleterious, distant, nonrenal organ dysfunctions is not well understood. Nonetheless, uremic toxins are thought to be one of the key mediators affecting organ dysfunction. ${ }^{10-12}$ Uremic toxins can be subdivided into three major groups based upon their chemical and physical characteristics: i) small, water-soluble, non-protein-bound compounds, such as urea; ii) small, lipid-soluble and/or protein-bound compounds, such as indoxyl sulfate (IS) and p-cresyl sulfate; iii) larger so-called middle-molecules, such as $\beta 2$-microglobulin. ${ }^{3-4}$

IS, a putative low-molecular weight uremic toxin, is excreted in the urine under normal kidney function, but is one of a representative group of sulfate-conjugated metabolites that are retained in the circulatory system during renal dysfunction. ${ }^{3,11,13}$ Serum IS levels are markedly elevated in patients undergoing dialysis treatment, with maximum serum concentrations of $>900 \mu \mathrm{M} .^{3}$ Previous studies demonstrate that IS induces oxidative stress in the kidney and cardiovascular system. ${ }^{5,6,14}$ For example, IS is one of the most powerful inducers of free radicals among various low-molecular weight uremic toxins..$^{5,6}$ Indeed, cytotoxic effects induced by IS can be explained by dysregulated oxygen metabolism and induction of oxidative stress in renal proximal tubular cells. ${ }^{14}$ IS is exclusively generated in the liver. Dietary protein-derived tryptophan is first metabolized to indole by tryptophanase of intestinal bacteria such as Escherichia coli. ${ }^{5,11}$ Following intestinal absorption, indole is hydroxylated to indoxyl by cytochrome P450 (CYP) 2E1 or CYP2A6, and subsequently conjugated to IS by sulfotransferase (SULT) 1A1 in the liver. ${ }^{15,16}$ IS generated in the liver enters the blood circulation and is efficiently taken up by renal proximal tubular cells via basolateral membrane-localized organic anion transporters, OAT1/SLC22A6 and OAT3/SLC22A8, before being excreted into the urine via unidentified apical membrane-localized transporters. ${ }^{17,18}$

Previously, we used experimental rats with cisplatininduced AKI to demonstrate oral administration of AST-120 (Kremezin ${ }^{\mathrm{TM}}$; Mitsubishi Tanabe Pharma Corporation, Osaka, Japan), a charcoal adsorbent for intestinal organic compounds that accumulates in patients with $\mathrm{CKD},{ }^{6}$ suppresses serum and renal accumulation of IS as well as significantly reducing the onset of AKI. ${ }^{19,20}$ Based on these findings, we proposed that low-molecular weight uremic toxins, which can be removed by AST- 120 treatment, contribute to the progression of kidney diseases in not only CKD but also in the development or derangement of AKI. Moreover, we found that phytochemical polyphenol compounds such as resveratrol and quercetin, which show a potent inhibitory effect against SULT1A1, significantly inhibited in vitro hepatic production of IS. ${ }^{21}$ These phytochemicals displayed a nephropreventive effect in cisplatin-induced AKI rats in association with a decreased accumulation of IS in serum, kidney, and liver. We suggested that agents with a potential inhibitory effect on hepatic IS production could be useful for preventing the progression of cisplatin-induced AKI. ${ }^{21}$ The pathophysiological role of IS in ischemic AKI has not yet been determined. In the present study we have examined the effect of meclofenamate, a potential SULT inhibitor, on IS accumulation and the development of kidney dysfunction in ischemic AKI rats.

\section{Materials and methods Chemicals}

IS was obtained from Sigma-Aldrich Co (St Louis, MO, USA). Apigenin, boldine, catechin-(+,-)-hydrate, diclofenac sodium, esculetin, graveoline, meclofenamic acid, myricetin, and naringenin were obtained from Meiji Seika Pharma Co, (Tokyo, Japan). Indole, carboxymethyl cellulose (CMC), methanol and nitric acid $\left(\mathrm{HNO}_{3}\right)$ were obtained from Wako Pure Chemical Industries, Ltd (Osaka, Japan). All chemicals used in this study were of analytical grade and commercially available.

\section{Inhibition experiment of hepatic IS production by rat liver $\$ 9$ fraction}

Rat liver S9 fraction was prepared according to our previous report with some modifications. ${ }^{21}$ The liver S9 fraction contains cytosol and microsomes. The microsomal component of the S9 fraction contains CYP isoforms and other enzymes 
mediating phase I metabolism. The cytosolic component includes transferases mediating phase II conjugation, including SULT. Liver was harvested from male Sprague Dawley rats at 6 weeks of age (Clea Japan, Inc., Tokyo, Japan). The liver homogenate in $50 \mathrm{mM}$ sodium phosphate buffer ( $\mathrm{pH}$ 7.4) containing $150 \mathrm{mM}$ potassium chloride was centrifuged at 9,000 $\mathrm{g}$ for 20 minutes at $4^{\circ} \mathrm{C}$, and the supernatant was used as the $\mathrm{S} 9$ containing microsomal fraction with CYP and cytosol fraction with SULT. The reaction mixture (total volume $250 \mu \mathrm{L}$ ) comprised $50 \mathrm{mM}$ sodium phosphate buffer ( $\mathrm{pH} 7.4$ ) containing the $\mathrm{S} 9$ fraction $(5-10 \mathrm{mg}$ protein $/ \mathrm{mL}$ ), indole, reduced nicotinamide adenine dinucleotide phosphate (NADPH) (1 mM), adenosine 3'-phosphate 5'-phosphosulfate $(20 \mu \mathrm{M})$ and uridine diphosphate glucuronic acid $(1 \mathrm{mM})$ with or without test compounds. Incubation of the reaction mixture was performed in a shaking water bath maintained at $37^{\circ} \mathrm{C}$ for 30 minutes. The reaction was terminated by addition of $250 \mu \mathrm{L}$ of ice-cold methanol. The reaction mixture was then centrifuged at 9,000 $g$ for 10 minutes and the supernatant obtained was used for liquid chromatography (LC)/mass spectrometry (MS)/MS analysis of IS concentration.

\section{Animal experiments}

All procedures for animal experiments were approved by Kumamoto University ethical committee concerning animal experiments, and animals were treated in accordance with the Guidelines of the United States National Institutes of Health regarding the care and use of animals for experimental procedures, and the Guidelines of Kumamoto University for the care and use of laboratory animals. Male Sprague Dawley rats at 6 weeks of age were housed in a standard animal maintenance facility at a constant temperature $\left(22^{\circ} \mathrm{C} \pm 2{ }^{\circ} \mathrm{C}\right)$ and humidity (50\%-70\%) and a 12/12 hour light/dark cycle for about a week before the day of the experiment, with food and water available ad libitum. Rats were anesthetized using sodium pentobarbital (50 mg/kg intraperitoneally), and placed on a heating plate $\left(39^{\circ} \mathrm{C}\right)$ to maintain a constant temperature. All surgery was conducted under anesthesia with pentobarbital, and all efforts were made to minimize animal suffering. The kidneys of male Sprague Dawley rats at 6 weeks of age were exposed via midline abdominal incisions. Renal ischemia/ reperfusion (I/R) was induced by vascular clamps (AS ONE, Osaka, Japan) over both pedicles for 30 minutes, followed by release of the clamps as reported previously. ${ }^{22}$ Sham animals (control) underwent anesthesia, laparotomy, and renal pedicle dissection only. Rats were divided into three different groups as follows: sham-operated rats (control rats), saline-administered rats with $\mathrm{I} / \mathrm{R}$ treatment, and meclofenamate-administered rats with $\mathrm{I} / \mathrm{R}$ treatment. Meclofenamate $(10 \mathrm{mg} / \mathrm{kg})$ was intravenously administered to rats 6,12 , and 18 hours after renal I/R treatment. Blood was collected 48 hours after I/R treatment from the abdominal aorta and centrifuged at 3,000 $g$ for 10 minutes to obtain the serum sample. Acetonitrile $(100 \mu \mathrm{L})$ was added to serum $(25 \mu \mathrm{L})$ and the mixture was centrifuged at $9,000 \mathrm{~g}$ for 10 minutes at $4^{\circ} \mathrm{C}$. The obtained supernatant $(50 \mu \mathrm{L})$ was diluted with LC/MS/MS mobile phase solution $(300 \mu \mathrm{L})$ and centrifuged at $9,000 \mathrm{~g}$ for 10 minutes at $4^{\circ} \mathrm{C}$. The supernatant was used for LC/MS/MS determination of IS concentration. Kidney was harvested 48 hours after I/R treatment and homogenized in phosphate-buffered saline $(\mathrm{pH}$ 7.4) using a Polytron PT3000 (Kinematica AG, Lucerne, Switzerland). After centrifugation at $3,000 \mathrm{~g}$ for 10 minutes at $4^{\circ} \mathrm{C}$, the obtained supernatant was used for LC/MS/MS assay of IS concentration. Levels of SCr (enzymatic method) and BUN (uricase ultraviolet [UV] method) were then measured.

\section{LC/MS/MS assay of IS concentration}

Aliquots of the extracts $(5 \mu \mathrm{L})$ were separated on a Symmetry ${ }^{\mathrm{TM}} \mathrm{C} 18(5 \mu \mathrm{m}, 3.9 \times 150 \mathrm{~mm}$ ) column (Waters Corp, Milford, MA, USA) interfaced with API3200 ${ }^{\mathrm{TM}} \mathrm{LC} / \mathrm{MS} / \mathrm{MS}$ system (AB SCIEX, Foster City, CA, USA) operating in negative TurboIonSpray mode. Samples were eluted at a flow rate of $0.2 \mathrm{~mL} / \mathrm{min}$ using a mobile phase consisting of $10 \mathrm{mM}$ ammonium acetate:acetonitrile $(73: 27 \mathrm{v} / \mathrm{v})$ at $40^{\circ} \mathrm{C}$. Relevant MS/MS settings were: charged aerosol detection gas at 3.0 psig; curtain at 40 psig; Ion Source Gas (GS) 1 at 50 psig; GS2 at $30 \mathrm{psig}$; IS at $-4500 \mathrm{~V}$ and temperature at $500^{\circ} \mathrm{C}$.

\section{Western blot analysis}

Western blot analysis for organic anion transporters, rOAT1 and rOAT3, was performed according to a previous report with some modifications. ${ }^{21,22}$ Briefly, kidneys were homogenized in an ice-cold homogenization buffer consisting of $230 \mathrm{mM}$ sucrose, $5 \mathrm{mM}$ Tris (hydroxymethyl) aminomethane hydrochloride (Tris- $\mathrm{HCl}$ ) (pH 7.5), 2 mM ethylenediaminetetraacetic acid, $0.1 \mathrm{mM}$ phenylmethanesulfonyl fluoride, $1 \mu \mathrm{g} / \mathrm{mL}$ leupeptin, and $1 \mu \mathrm{g} / \mathrm{mL}$ pepstatin $\mathrm{A}$. After measuring protein content using a bicinchoninic acid (BCA) protein assay reagent (Thermo Fisher Scientific, Waltham, MA, USA), each sample was mixed in loading buffer $(2 \mathrm{w} / \mathrm{v} \%$ sodium dodecyl sulfate [SDS], $125 \mathrm{mM}$ Tris- $\mathrm{HCl} \mathrm{pH} 7.2,20 \mathrm{v} / \mathrm{v} \%$ glycerol, and $5 \mathrm{v} / \mathrm{v} \% 2$-mercaptoethanol) and heated at $95^{\circ} \mathrm{C}$ for 2 minutes. The samples were subjected to sodium dodecyl sulfate-polyacrylamide gel electrophoresis using a 7.5\% gel, and transferred onto a polyvinylidene difluoride membrane (Immobilon-P; EMD Millipore, Billerica, MA, USA) by semi- 
dry electroblotting. The membrane was blocked overnight at $4{ }^{\circ} \mathrm{C}$ with $2 \mathrm{v} / \mathrm{v} \%$ ECL Advance Blocking Agent (GE Healthcare UK Ltdss, Little Chalfont, UK) in $50 \mathrm{mM}$ Trisbuffered saline ( $\mathrm{pH} 7.6$ ) containing $0.3 \mathrm{v} / \mathrm{v} \%$ Tween 20 , and then incubated for 1 hour at room temperature with a primary antibody specific for rOAT1 or rOAT3 (gift from Dr Inui, Kyoto University Hospital). The blots were then washed with Tris-buffered saline containing Tween 20 before incubation with the secondary antibody (horseradish peroxidase-linked anti-rabbit immunoglobulin $\mathrm{F}(\mathrm{ab}) 2$ or horseradish peroxidaselinked anti-mouse immunoglobulin F(ab)2) (GE Healthcare UK Ltd) for 1 hour at room temperature. Immunoblots were visualized with an ECL system (ECL Advance Western Blotting Detection Kit; GE Healthcare UK Ltd).

\section{Measurement of prostaglandin (PG) E2 concentration in kidneys}

Kidney was harvested 48 hours after I/R treatment and homogenized in $100 \mathrm{mM}$ phosphate buffer containing $1 \mathrm{mM}$ EDTA and $10 \mu \mathrm{M}$ COX inhibitor ${ }^{\mathrm{TM}}(\mathrm{pH}$ 7.4) using a Polytron PT3000 (Kinematica AG). After centrifugation at $8,000 \mathrm{~g}$ for 10 minutes at $4^{\circ} \mathrm{C}$, the obtained supernatant was used to assay prostaglandin E2 (PGE2) concentration using a Prostaglandin E2 Express EIA Kit (Cayman Chemical Co, San Diego, CA, USA).

\section{Statistical analysis}

Data were analyzed statistically by analysis of variance, followed by Scheffé's multiple comparison test. A $P$-value of $<0.05$ was considered statistically significant. All data are represented as the mean \pm standard deviation (SD).

\section{Results}

\section{In vitro inhibitory effects of several compounds on IS production using the rat hepatic $S 9$ fraction}

In our previous study, ${ }^{21}$ we found that quercetin, curcumin, and resveratrol, well-known potent inhibitors of SULT, showed strong inhibitory effects on IS production. The apparent inhibition constant $(\mathrm{Ki})$ values of quercetin, curcumin, and resveratrol for IS production were 1.0, 1.8, and $1.5 \mu \mathrm{M}$, respectively. ${ }^{23}$ By contrast, the apparent Ki values of tranylcypromine, a CYP2A6 inhibitor, and diethyldithiocarbamate, a CYP2E1 inhibitor, were 148 and $1,859 \mu \mathrm{M}$, respectively. ${ }^{21}$ Based on these findings, we further examined the inhibitory potencies of several compounds, including other phytochemical polyphenols and clinically used drugs (chemical structures shown in Figure 1), on hepatic IS production by using the liver S9 fraction. Among the compounds examined, polyphenol derivatives showed relatively potent inhibitory effects on hepatic IS production; percentage of inhibition, esculetin $(84.9 \% \pm 0.3 \%)$, apigenin $(84.2 \% \pm 3.3 \%)$, and myricetin $(75.6 \% \pm 1.0 \%)$ (Figure 2$)$. Meclofenamate, a nonsteroidal anti-inflammatory drug, also exhibited strong inhibition $(83.3 \% \pm 1.9 \%)$ of IS production. Another non-steroidal antiinflammatory drug, diclofenac, showed a moderate inhibitory effect $(31.8 \% \pm 8.8 \%)$. The apparent inhibitory constant $\left(\mathrm{IC}_{50}\right)$ values for these compounds on IS production were kinetically determined based on the data of concentration-dependent inhibition experiments (Figure 3). Among these compounds, meclofenamate showed the most potent inhibitory effect on IS production (apparent $\mathrm{IC}_{50}$ of $1.34 \mu \mathrm{M}$ ).

\section{Effect of meclofenamate on IS concentrations in rats treated with renal $I / R$}

The effect of meclofenamate on IS concentrations in serum, kidney, and liver was examined in rats treated with renal $\mathrm{I} / \mathrm{R}$. Meclofenamate was administered three times to rats by intravenous injection at a dose of $10 \mathrm{mg} / \mathrm{kg}$. Serum IS concentration was markedly increased in $\mathrm{I} / \mathrm{R}$ rats 48 hours after surgery compared to that in control rats (Figure 4). However, meclofenamate administration significantly suppressed this increase in serum IS concentration (Figure 4). Furthermore, the IS concentration in both kidney and liver was dramatically elevated after renal I/R treatment, but this elevation in IS level in these organs was significantly reduced by administration of meclofenamate (Figure 4).

\section{Effect of meclofenamate on renal function of rats treated with renal I/R}

Figure 5 illustrates $\mathrm{SCr}$ and BUN levels in rats treated with renal I/R. SCr and BUN were markedly elevated by renal $\mathrm{I} / \mathrm{R}$ treatment, but these increases were significantly restored by the administration of meclofenamate, suggesting this compound elicits a nephropreventing effect.

\section{Effect of meclofenamate on the expression of rOATI and rOAT3 and the level of PGE2 in an ischemic kidney}

In our previous study, we reported that expression of organic anion transporters rOAT1 and rOAT3, located on the basolateral membrane of renal proximal tubular cells, was markedly downregulated after renal I/R treatment in rats. ${ }^{22}$ As shown in Figure 6, the expression levels of both rOAT1 
<smiles>O=c1cc(-c2ccc(O)cc2)oc2cc(O)cc(O)c12</smiles>

Diclofenac sodium<smiles>O=C(Cc1ccccc1Nc1c(Cl)cccc1Cl)O[Na]</smiles><smiles>O=C1C[C@@H](c2ccc(O)cc2)Oc2cc(O)cc(O)c21</smiles><smiles>COc1cc2c(cc1O)C[C@H]1c3c(cc(O)c(OC)c3-2)CCN1C</smiles>

Esculetin<smiles>O=c1ccc2cc(O)c(O)cc2o1</smiles>

Meclofenamic acid

$\mathrm{Na}^{+}$<smiles>Cc1ccc(Cl)c(Nc2ccccc2C(=O)[O-])c1Cl</smiles>

Catechin-(+,-)hydrate<smiles>Oc1cc(O)c2c(c1)OC(c1ccc(O)c(O)c1)C(O)C2</smiles>

Graveoline<smiles>Cn1c(-c2ccc3c(c2)OCO3)cc(=O)c2ccccc21</smiles>

Myricetin<smiles>O=c1c(O)c(-c2cc(O)c(O)c(O)c2)oc2cc(O)cc(O)c12</smiles>

Figure I Chemical structures of compounds examined for their inhibitory effect on hepatic IS production. Abbreviation: IS, indoxyl sulfate.

and rOAT3 were markedly decreased in the kidney of $\mathrm{I} / \mathrm{R}$-treated rats. However, the expression levels of these two transporters were largely restored by administration of meclofenamate.

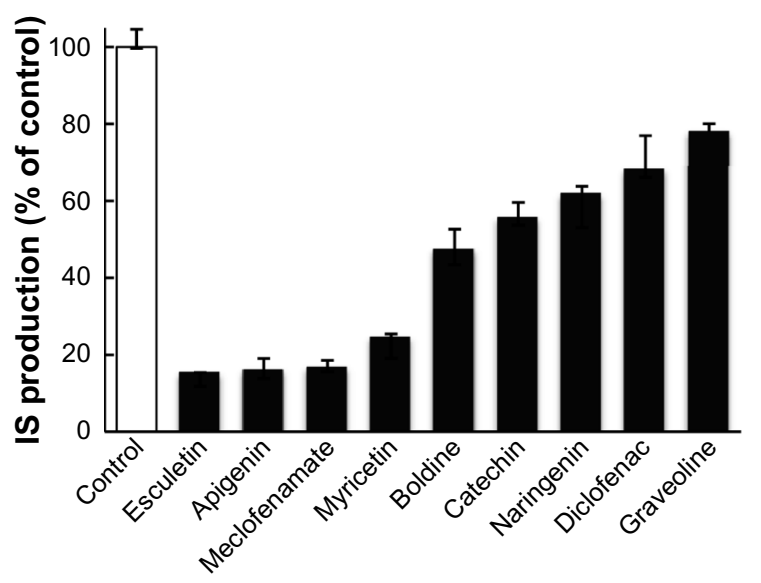

Figure $\mathbf{2}$ In vitro inhibitory effect of several compounds on IS production using the rat liver $\mathbf{S} 9$ fraction.

Notes: The reaction mixture (total volume $250 \mu \mathrm{L}$ ), containing $50 \mathrm{mM}$ sodium phosphate buffer $(\mathrm{pH} 7.4)$, rat liver $\mathrm{S} 9$ fraction $(5-10 \mathrm{mg}$ protein $/ \mathrm{mL})$, indole (100 $\mu \mathrm{M})$, NADPH (I mM), adenosine 3 '-phosphate $5^{\prime}$-phosphosulfate $(20 \mu \mathrm{M})$, and uridine diphosphate glucuronic acid ( $\mathrm{mM})$, was incubated with or without test compound $(10 \mu \mathrm{M})$ at $37^{\circ} \mathrm{C}$ for 30 minutes. Each column represents the mean \pm standard deviation for three independent measurements.

Abbreviations: NADPH, nicotinamide adenine dinucleotide phosphate; IS, indoxyl sulfate.
PGE2 can be transiently produced by an increased COX2 activity in response to oxidative stress. In the ischemic kidney of rats, PGE2 is reported to induce downregulation of rOAT1 and rOAT3. ${ }^{23}$ Thus, the PGE2 level in rats after renal I/R treatment was assayed. Renal I/R treatment caused a marked elevation in the level of PGE2, but this increase was prevented partially by administration of meclofenamate compared to that of control (sham) (Figure 7).

\section{Discussion}

Based on an in vitro IS production assay using rat liver S9 fraction, we found that phytochemical polyphenols showed a relatively potent inhibitory effect on hepatic IS production. Among the compounds tested, esculetin, apigenin, and myricetin displayed strong inhibitory effects on IS production (Figure 2). In our previous study, the inhibitors of SULT activity, such as resveratrol, quercetin, and curcumin, showed potent inhibitory effects on IS production compared with the inhibitors of CYP2E1 and CYP2A6, including diethyldithiocarbamate or tranylcypromine. ${ }^{21}$ These results suggested that SULT-mediated sulfation of indoxyl could be the inhibitor-sensitive reaction in hepatic IS production. Flavonoid compounds have been reported to inhibit 

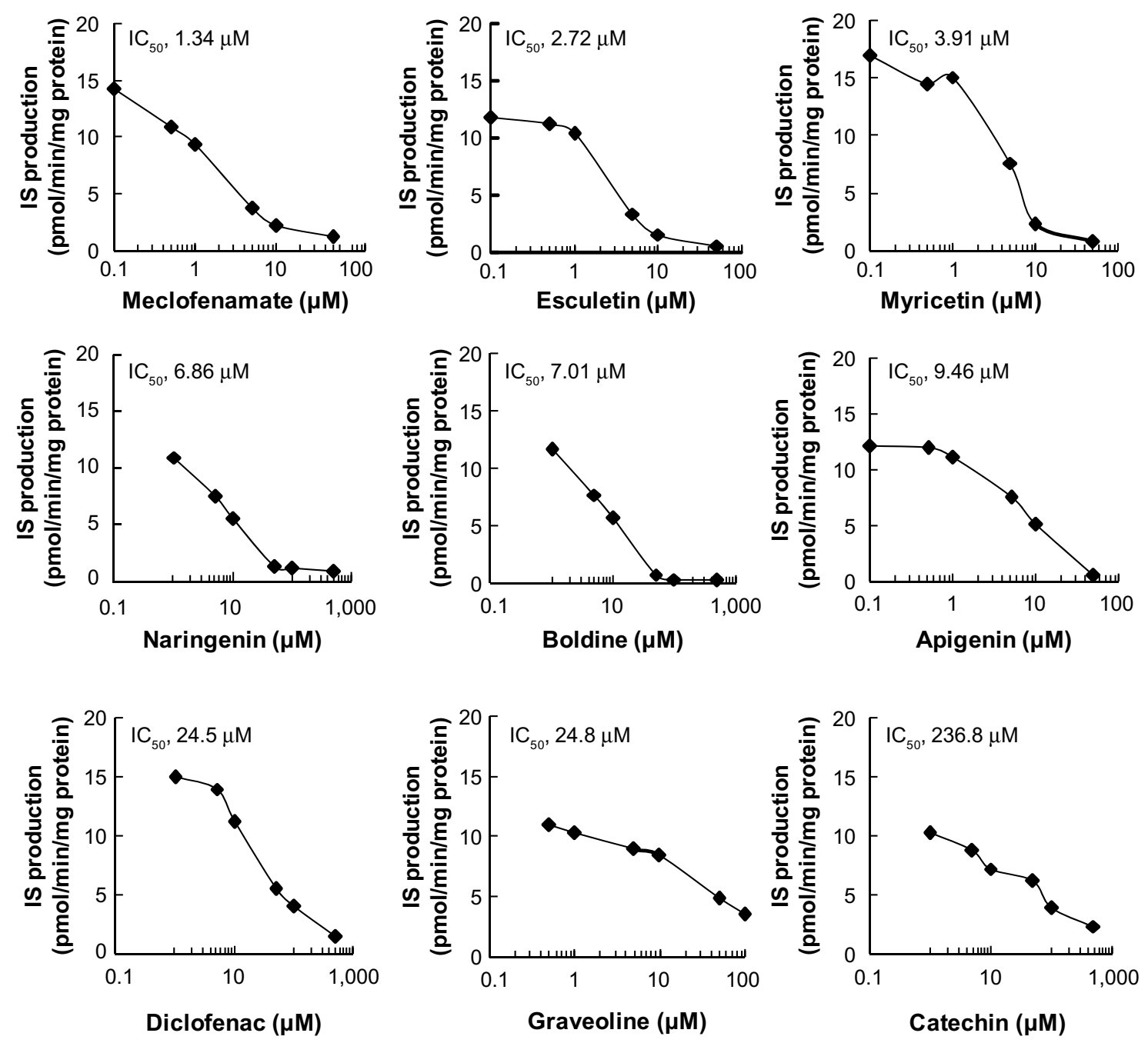

Figure 3 Dose-dependent inhibition of IS production by several compounds using rat liver S9 fraction.

Notes: The reaction mixture containing $50 \mathrm{mM}$ sodium phosphate buffer ( $\mathrm{pH} 7.4$ ), rat liver S9 fraction, indole (I00 $\mu M)$, NADPH (I mM), adenosine $3^{\prime}$-phosphate $5^{\prime}$ phosphosulfate $(20 \mu \mathrm{M})$, and uridine diphosphate glucuronic acid $(\mathrm{I} \mathrm{mM})$ was incubated at $37^{\circ} \mathrm{C}$ for 30 minutes with or without test compounds at various concentrations. Each point represents the mean of three independent measurements. The apparent $I_{50}$ values are indicated for each graph for compound tested.

Abbreviations: IS, indoxyl sulfate; NADPH, nicotinamide adenine dinucleotide phosphate.

SULT activity. ${ }^{24}$ Apigenin and myricetin were reported to have potent inhibitory effects on CYP3A4 and CYP2C9. ${ }^{25,26}$ In addition, myricetin and apigenin were found to be potent inhibitors of phenolsulfotransferase-mediated sulfoconjugation of acetaminophen and minoxidil with $\mathrm{IC}_{50}$ values $<1 \mu \mathrm{M}$ or resveratrol with $\mathrm{IC}_{50}$ values $<3 \mu \mathrm{M}{ }^{27,28}$ The inhibitory potency of esculetin against CYP or SULT enzymes has not been reported. Based on these findings, the observed inhibition of hepatic IS production by esculetin, myricetin, and apigenin could predominantly depend on inhibition of SULT activity rather than CYP2E1 or CYP2A6.

Meclofenamate, a non-polyphenolic and non-steroidal anti-inflammatory drug, also exhibited a strong inhibition of hepatic IS production with an apparent $\mathrm{IC}_{50}$ of $1.3 \mu \mathrm{M}$.
Previous reports suggest that non-steroidal anti-inflammatory agents including meclofenamate, nimesulide, piroxicam, and aspirin competitively inhibit human phenol SULT with $\mathrm{IC}_{50}$ values ranging from $0.1 \mu \mathrm{M}$ to $3,800 \mu \mathrm{M} .{ }^{29}$ In addition, among non-steroidal anti-inflammatory drugs, fenamic compounds including mefenamic acid, flufenamic acid, niflumic acid, and meclofenamic acid were reported to impair human liver SULT activity without phenolic hydroxyl group in their molecules. ${ }^{30}$ However, the effect of meclofenamate on SULT-mediated IS production was not examined. We previously reported that oral administration of phytochemical polyphenols showed a nephropreventive effect on cisplatin-induced AKI in rats. ${ }^{21}$ These effects were associated with a significant suppression of serum and renal accumulation of IS. Thus, the potent inhibitory 
A

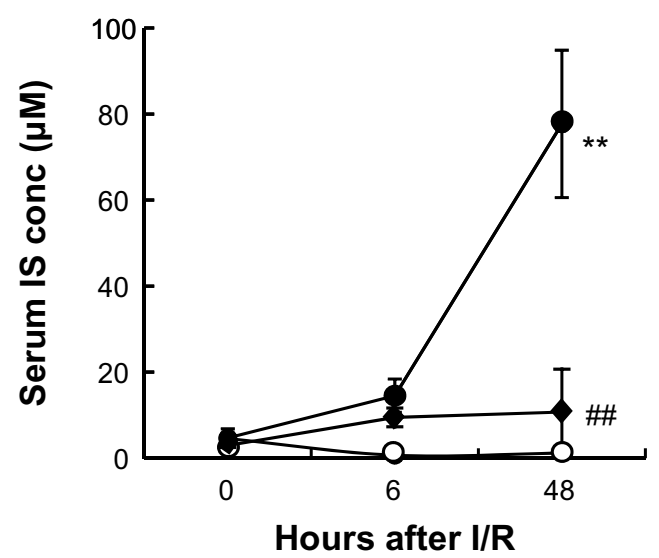

C

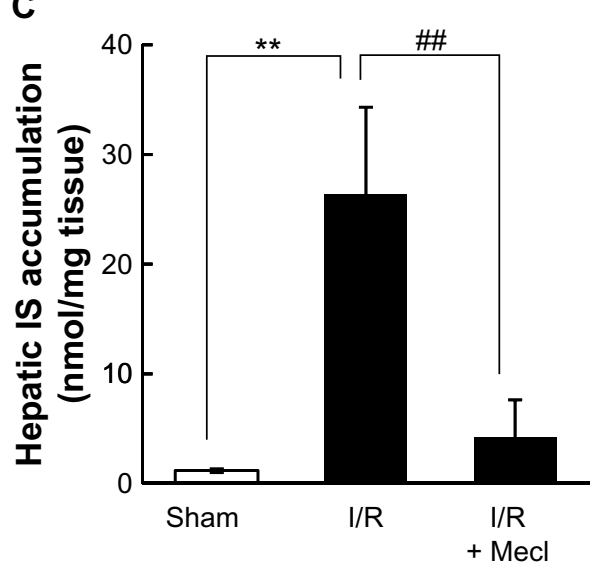

B

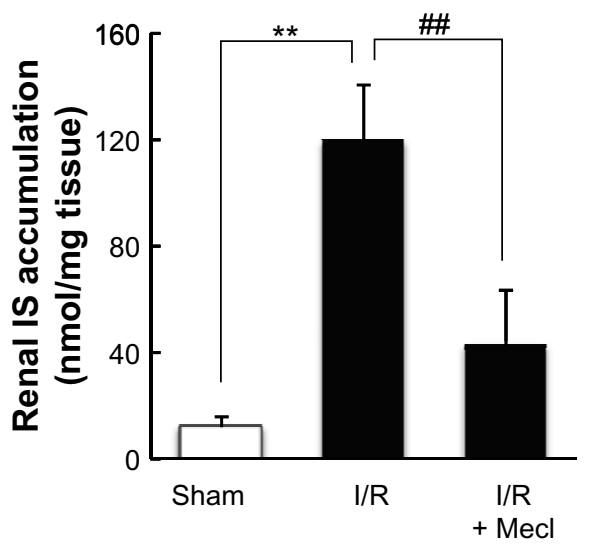

Figure 4 Effect of meclofenamate on IS concentration in rats treated with renal I/R.

Notes: (A) Serum IS concentration in control rats (open circle) and renal I/R-treated rats with (closed rhombus) or without (closed circle) intravenous administration of meclofenamate $(10 \mathrm{mg} / \mathrm{kg}) 6,12$, and 18 hours after renal I/R treatment. Each point represents the mean \pm standard deviation for three to four rats in each group. Renal (B) and hepatic (C) IS concentration in control rats (sham) and renal I/R-treated rats with $(I / R+M e c l)$ or without (I/R) intravenous administration of meclofenamate. $* * P<0.0 I$ versus sham (control); ${ }^{\#} P<0.0$ I versus I/R.

Abbreviations: IS, indoxyl sulfate; I/R, ischemia/reperfusion; Mecl, meclofenamate.

A

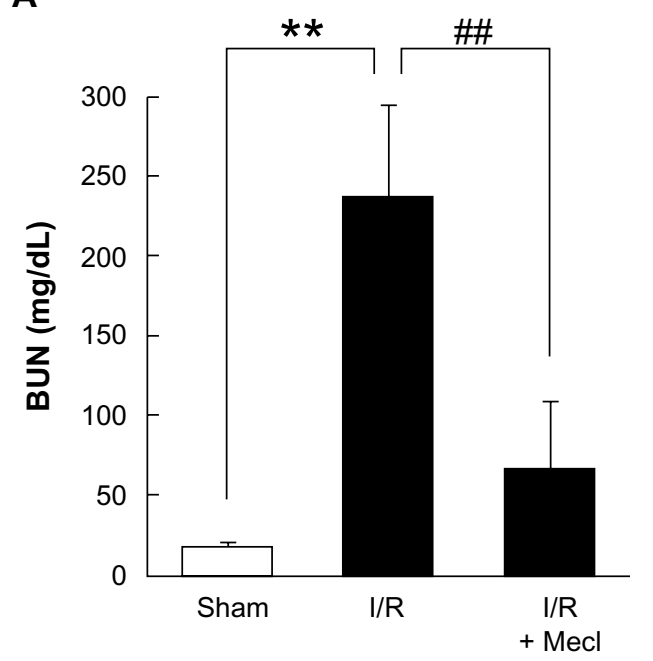

B

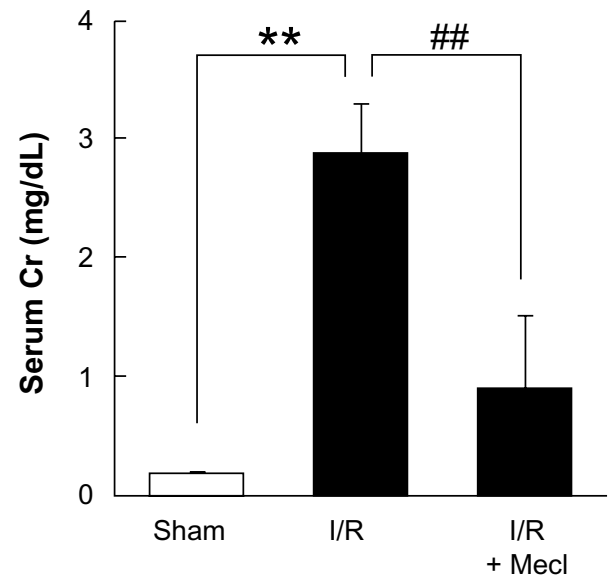

Figure 5 Effect of meclofenamate on renal function of rats treated with renal I/R.

Notes: Serum BUN $(\mathbf{A})$ and creatinine $(\mathbf{B})$ levels in control rats (sham), renal I/R-treated rats with (I/R + Mecl) or without (I/R) intravenous administration of meclofenamate. $* * P<0.0$ I versus sham (control); $P<0.0$ I versus I/R.

Abbreviations: I/R, ischemia/reperfusion; BUN, blood urea nitrogen; Mecl, meclofenamate; Cr, creatinine. 
A
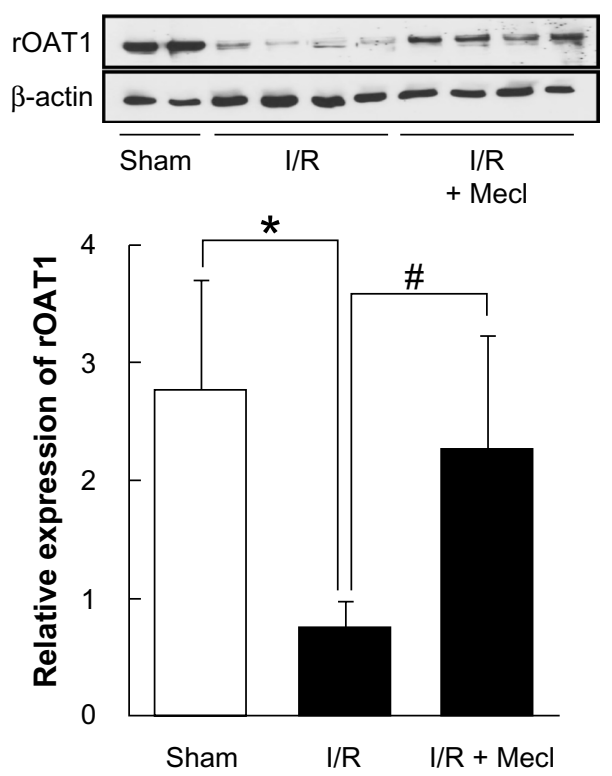

B
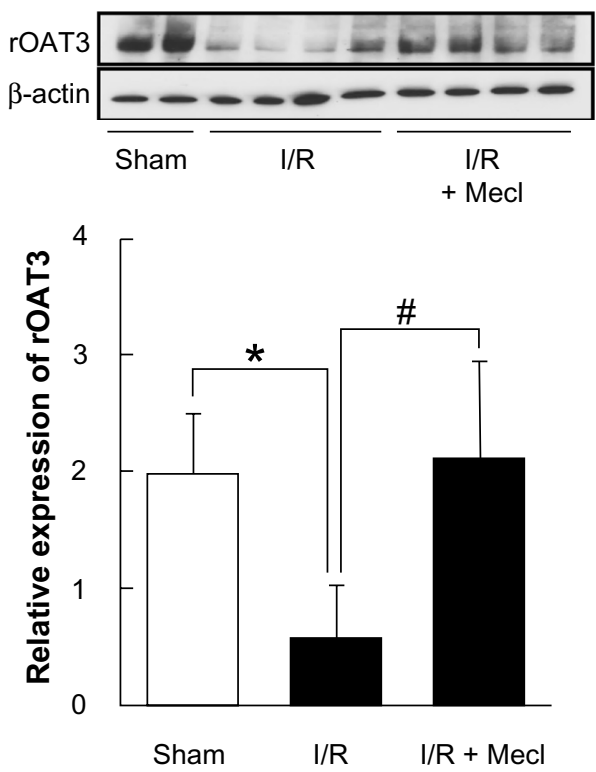

Figure 6 Expression of rOATI and rOAT3 in the kidney of rats treated with I/R. Meclofenamate $(10 \mathrm{mg} / \mathrm{kg})$ was intravenously administered to rats 6 , 12 , and 18 hours after renal I/R.

Notes: Renal tissue samples were collected 48 hours after I/R treatment, and used for immunoblotting of rOATI and rOAT3. Panels (A and B) show the representative blots for rOATI and rOAT3, respectively. The band density of rOATI and rOAT3 was determined densitometrically and the relative density ratio to control (sham) is represented as columns with the mean \pm standard deviation for three to four rats in each group. $* P<0.05$ versus control (sham); ${ }^{*} P<0.05$ versus $I / R$.

Abbreviations: rOAT, rat organic anion transporter; I/R, ischemia/reperfusion; Mecl, meclofenamate.

effect of meclofenamate on IS production encouraged us to examine its in vivo effects on IS accumulation and ischemic AKI in rats. An increase in IS concentration in the kidney and liver of I/R-treated rats was largely blocked by the intravenous

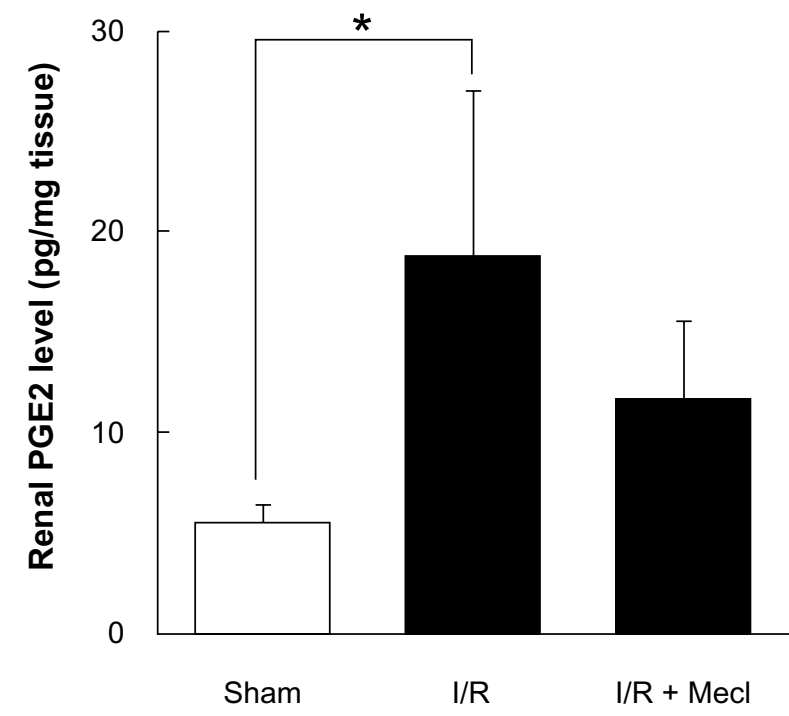

Figure 7 Renal PGE2 levels in the kidney of rats.

Notes: Effect of meclofenamate on PGE2 level was examined in the kidney of control rats (sham) and renal I/R-treated rats with $(I / R+M e c l)$ or without $(I / R)$ intravenous administration of meclofenamate $(10 \mathrm{mg} / \mathrm{kg})$. Each column represents the mean \pm standard deviation for three to four rats in each group. $* P<0.05$ versus control (sham). Abbreviations: PGE2, prostaglandin E2; I/R, ischemia/reperfusion; Mecl, meclofenamate. administration of meclofenamate (see Figures 4 and 5). Furthermore, treatment with meclofenamate significantly restored the increased levels of SCr and BUN in I/R-treated rats (Figure 5). The administration of meclofenamate was associated with the restoration of renal basolateral membrane OATs mediating cellular accumulation of IS, rOAT1, and rOAT3, which were markedly downregulated by renal $\mathrm{I} / \mathrm{R}$ treatment (Figure 6). Sauvant et al demonstrated that PGE2 leads to downregulation of both rOAT1 and rOAT3 in the rat proximal tubular cell line NRK-52E after long-term exposure. ${ }^{31}$ Furthermore, PGE2 levels have been reported to increase in the kidney cortex after acute renal ischemia ${ }^{32,33}$ or during chronic renal ischemia. ${ }^{34}$ Notably, Schneider et al reported that administration of a low-dose of indomethacin, a non-steroidal anti-inflammatory agent, after renal I/R treatment prevented I/R-induced downregulation of rOAT1 and rOAT3 during reperfusion and elicited a nephropreventive effect on kidney function..$^{23}$ They concluded that a low-dose of indomethacin $(1 \mathrm{mg} / \mathrm{kg})$ applied directly after ischemia has a beneficial effect on renal outcome after ischemia, and that PGE2 plays an important role in the development or maintenance of renal damage. Pharmacological intervention of COX activity may be a therapeutically relevant option after acute ischemic injury of the kidney. ${ }^{23}$ Based on these findings and the present results, the restorative effect of meclofenamate 
on rOAT1/3 may be caused by suppressing COX2-mediated PGE2 production in the kidney. The substantial restoration or maintenance of these transporters should enhance renal excretion of IS, thereby reducing IS-induced cytotoxicity in the kidney. Thus, meclofenamate could have two pathways for its nephropreventive effect in ischemic AKI: i) the potent inhibition of hepatic IS production followed by suppression of IS accumulation in serum and kidney, and ii) the prevention of renal COX2-mediated PGE2 production, that could be induced by renal I/R treatment and/or IS accumulation in the kidney, followed by secondary restoration of OATs' expression.

\section{Conclusion}

Meclofenamate inhibits hepatic production of IS, which results in decreased serum and renal accumulation of IS, and protects I/R-induced downregulation of rOAT1 and rOAT3 expression via COX2-mediated PGE2 production, thereby eliciting a nephropreventive effect in ischemic AKI. These findings will help in the development of novel therapeutic drugs that prevent AKI.

\section{Acknowledgments}

This work was supported by Japan Society for the Promotion of Science (JSPS) KAKENHI (25293040 grant to HS); A-STEP (Adaptable and Seamless Technology Transfer Program through Target-driven R\&D) (AS242Z02505Q grant to HS); Japan Science and Technology Agency (JST) Accelerating Utilization of University IP Program (FY2013-300-148007 grant to HS); Center for Clinical and Translational Research Kyushu University Hospital (Seeds A29 grant to HS).

\section{Author contributions}

HS contributed to the conception and design of the work. All authors contributed toward data analysis, drafting, and revising the manuscript.

\section{Disclosure}

The authors report no conflicts of interest in this work.

\section{References}

1. Cruz DN, Ricci Z, Ronco C. Clinical review: RIFLE and AKIN - time for reappraisal. Crit Care. 2009;13(3):211-219.

2. Murray PT, Devarajan P, Levey AS, et al. A framework and key research questions in AKI diagnosis and staging in different environments. Clin J Am Soc Nephrol. 2008;3(3):864-868.

3. Vanholder R, De Smet R, Glorieux G, et al. Review on uremic toxins: classification, concentration, and interindividual variability. Kidney Int. 2003;63(5):1934-1943.

4. Neirynck N1, Vanholder R, Schepers E, et al. An update on uremic toxins. Int Urol Nephrol. 2013;45(1):139-150.
5. Niwa T. Indoxyl sulfate is a nephro-vascular toxin. J Ren Nutr. 2010; 20(Suppl 5):S2-S6.

6. Niwa T. Role of indoxyl sulfate in the progression of chronic kidney disease and cardiovascular disease: experimental and clinical effects of oral sorbent AST-120. Ther Apher Dial. 2011;15(2):120-124.

7. Vincent JL. Acute kidney injury, acute lung injury and septic shock: how does mortality compare? Contrib Nephrol. 2011;174:71-77.

8. Liu KD, Matthay MA. Advances in critical care for the nephrologist: acute lung injury/ARDS. Clin J Am Soc Nephrol. 2008;3(2):578-586.

9. May CN, Calzavacca P, Ishikawa K, et al. Novel targets for sepsisinduced kidney injury: the glomerular arterioles and the sympathetic nervous system. Exp Physiol. 2012;97(11):1168-1177.

10. Herget-Rosenthal S, Glorieux G, Jankowski J, Jankowski V. Uremic toxins in acute kidney injury. Semin Dial. 2009;22(4):445-448.

11. Saito H. Toxico-pharmacological perspective of the Nrf2-Keap1 defense system against oxidative stress in kidney diseases. Biochem Pharmacol. 2013;85(7):865-872.

12. Bagshaw SM, Hoste EA, Braam B, et al. Cardiorenal syndrome type 3 : pathophysiologic and epidemiologic considerations. Contrib Nephrol. 2013;182:137-157.

13. Vanholder R, Schepers E, Pletinck A, Neirynck N, Glorieux G. An update on protein-bound uremic retention solutes. J Ren Nutr. 2012;22(1):90-94.

14. Chiang CK, Tanaka T, Nangaku M. Dysregulated oxygen metabolism of the kidney by uremic toxins: review. J Ren Nutr. 2012;22(1):77-80.

15. Banoglu E, King RS. Sulfation of indoxyl by human and rat aryl (phenol) sulfotransferases to form indoxyl sulfate. Eur J Drug Metab Pharmacokinet. 2002;27(2):135-140.

16. Banoglu E, Jha GG, King RS. Hepatic microsomal metabolism of indole to indoxyl, a precursor of indoxyl sulfate. Eur J Drug Metab Pharmacokinet. 2001;26(4):235-240.

17. Enomoto A, Takeda M, Tojo A, et al. Role of organic anion transporters in the tubular transport of indoxyl sulfate and the induction of its nephrotoxicity. J Am Soc Nephrol. 2002;13(7):1711-1720.

18. Miyazaki T, Ise M, Hirata M, et al. Indoxyl sulfate stimulates renal synthesis of transforming growth factor-beta 1 and progression of renal failure. Kidney Int Suppl. 1997;63:S211-S214.

19. Iwata K, Watanabe H, Morisaki T, et al. Involvement of indoxyl sulfate in renal and central nervous system toxicities during cisplatin-induced acute renal failure. Pharm Res. 2007;24(4):662-671.

20. Morisaki T, Matsuzaki T, Yokoo K, et al. Regulation of renal organic ion transporters in cisplatin-induced acute kidney injury and uremia in rats. Pharm Res. 2008;25(11):2526-2533.

21. Kusumoto M, Kamobayashi H, Sato D, et al. Alleviation of cisplatininduced acute kidney injury using phytochemical polyphenols is accompanied by reduced accumulation of indoxyl sulfate in rats. Clin Exp Nephrol. 2011;15(6):820-830.

22. Matsuzaki T, Watanabe H, Yoshitome K, et al. Downregulation of organic anion transporters in rat kidney under ischemia/reperfusioninduced acute renal failure. Kidney Int. 2007;71(6):539-547.

23. Schneider R, Meusel M, Renker S, et al. Low-dose indomethacin after ischemic acute kidney injury prevents downregulation of Oat1/3 and improves renal outcome. Am J Physiol Renal Physiol. 2009;297(6):F1614-F1621.

24. Wong CC, Williamson G. Inhibition of hydroxycinnamic acid sulfation by flavonoids and their conjugated metabolites. Biofactors. 2013;39(6):644-651.

25. Kimura Y, Ito H, Ohnishi R, Hatano T. Inhibitory effects of polyphenols on human cytochrome P450 3A4 and 2C9 activity. Food Chem Toxicol. 2010;48(1):429-435.

26. Li C, Lim SC, Kim J, Choi JS. Effects of myricetin, an anticancer compound, on the bioavailability and pharmacokinetics of tamoxifen and its main metabolite, 4-hydroxytamoxifen, in rats. Eur J Drug Metab Pharmacokinet. 2011;36(3):175-182.

27. Eaton EA, Walle UK, Lewis AJ, et al. Flavonoids, potent inhibitors of the human P-form phenolsulfotransferase. Potential role in drug metabolism and chemoprevention. Drug Metab Dispos. 1996;24(2):232-237. 
28. De Santi C, Pietrabissa A, Spisni R, Mosca F, Pacifici GM. Sulphation of resveratrol, a natural compound present in wine, and its inhibition by natural flavonoids. Xenobiotica. 2000;30(9):857-866.

29. King RS, Ghosh AA, Wu J. Inhibition of human phenol and estrogen sulfotransferase by certain non-steroidal anti-inflammatory agents. Curr Drug Metab. 2006;7(7):745-753.

30. Vietri M, De Santi C, Pietrabissa A, et al. Fenamates and the potent inhibition of human liver phenol sulphotransferase. Xenobiotica. 2000;30(2):111-116.

31. Sauvant C, Holzinger H, Gekle M. Prostaglandin E2 inhibits its own renal transport by downregulation of organic anion transporters rOAT1 and rOAT3. J Am Soc Nephrol. 2006;17(1):46-53.
32. Myers SI, Wang L, Liu F, Bartula LL. Suprarenal aortic clamping and reperfusion decreases medullary and cortical blood flow by decreased endogenous renal nitric oxide and PGE2 synthesis. J Vasc Surg. 2005;42(3):524-531.

33. Tokuyama H, Hayashi K, Matsuda H, et al. Role of nitric oxide and prostaglandin E2 in acute renal hypoperfusion. Nephrology (Carlton). 2003;8(2):65-71.

34. Tokuyama H, Hayashi $\mathrm{K}$, Matsuda $\mathrm{H}$, et al. Stenosis-dependent role of nitric oxide and prostaglandins in chronic renal ischemia. Am J Physiol Renal Physiol. 2002;282(5):F859-F865.

\section{Publish your work in this journal}

Drug Design, Development and Therapy is an international, peerreviewed open-access journal that spans the spectrum of drug design and development through to clinical applications. Clinical outcomes, patient safety, and programs for the development and effective, safe, and sustained use of medicines are a feature of the journal, which has also been accepted for indexing on PubMed Central. The manuscript management system is completely online and includes a very quick and fair peer-review system, which is all easy to use. Visit http://www.dovepress.com/testimonials.php to read real quotes from published authors.

Submit your manuscript here: http://www.dovepress.com/drug-design-development-and-therapy-journal 